\title{
Integrated Analyses of miRNAome and Transcriptome Reveal The Function of miRNAs in Morphogenesis of Organs and Defense of Endophytes in Pseudostellaria Heterophylla
}

Jun Li ( $\square$ speker@163.com )

Guizhou University of Traditional Chinese Medicine

Chongmin Wang

Guizhou University of Traditional Chinese Medicine

Tao Zhou

Guizhou University of Traditional Chinese Medicine

Haijun Jin

Guizhou University of Traditional Chinese Medicine

Xiaoqing Liu

Guizhou University of Traditional Chinese Medicine

\section{Research Article}

Keywords: Pseudostellaria heterophylla, miRNAs, Correlation analysis, Morphogenesis of organs, Hormone signal transduction

Posted Date: March 1st, 2021

DOI: https://doi.org/10.21203/rs.3.rs-239755/v1

License: @ (1) This work is licensed under a Creative Commons Attribution 4.0 International License. Read Full License 


\section{Abstract}

Background: miRNAs play a crucial role in plant development and growth by inhibiting the function of targeted gene mRNAs at the post transcription level. However, none miRNAs in Pseudostellaria heterophylla has been reported and their function in morphogenesis of organs and continuous cropping obstacles is still unclear.

Results: A total of 163 conserved miRNAs (belonging to 66 families) and 303 level miRNAs were identified and some of them showed specifically up or down regulated expression in different tissues. Further, numbers of unigenes involved in Plant-pathogen interaction and MAPK signaling pathway-plant was targeted by miRNAs from $P$. heterophylla by using GO and KEGG analysis. Significant negative correlation between expression profiles of 30 miRNAs and their targets gene (37 unigenes) were observed. Further, a large number of genes involving with signal transduction of auxin, zeatin, abscisic acid and jasmonic acid were targeted by identified miRNAs in $P$. heterophylla. A predicated target gene of a conserved and novel miRNA was validated by $5^{\prime} R L M-R A C E$, respectively. A large number of mRNA from four kinds of endophytes was targeted by miRNAs of $P$. heterophylla, and most genes were targeted by miR414.

Conclusion: We report a population of $P$. heterophylla miRNAs from four different vegetative organs by high throughput sequencing, and analyzed combining with the constructed transcriptome. These results may help to explain the function of miRNA in morphogenesis of organs and defense of endophytes in $P$. heterophylla, and provide theoretical basis for breeding and genetic improvement of $P$. heterophylla.

\section{Background}

MicroRNA (miRNA) is a kind of endogenous single stranded non-coding small RNA [1], which inhibits the function of targeted gene mRNAs at the post transcription level by degrading or inhibiting the translation [2]. Since the first miRNA (lin 4) in plants was identified [3], a large amount of miRNAs have been discovered from various species. Recent evidence has indicated that miRNA not only can influent the growth and development of plants, the differentiation and morphogenesis of organs by directly regulating the target genes [4], but also participate in the signal transduction of plants [5]. miRNA can improve the tolerance to environmental stress by regulating the transcription factor[6], such as drought, low temperature, high salt and low nitrogen. Furthermore, miRNAs can also improve the resistance to biological stress of plants by inhibiting the gene expression of pathogenic microorganisms [7-8].

During the synthesis of miRNAs, primary miRNAs (pri-miRNA), an imperfect long folded back structure, are firstly transcribed by RNA polymerase II [9]. The pri-miRNAs is processed into 70-nt premiRNAs by Dicer-like protein (endoribonuclease having RNase III activity), in association with other protein factors (HYL1and others), and subsequently released as miRNA: miRNA* duplexes by different DCL protrein [10]. The 3' terminal of these duplexes are methylated by HEN1, which can prevent miRNA degradation and help the miRNA be incorporated into the silencing complex or effector complex (RISC-RNA Induced Silencing Complex) containing argonaute proteins [11-13]. Methylated miRNA: miRNA* duplexes are subsequently transported to the cytoplasm with the help of the HASTY protein [11, 14], while the other strand (miRNA* or passenger strand) gets degraded. The RISC-RNA induced silencing complex reduces the expression of target mRNA by guiding the cleavage of complementary target mRNAs and inhibit its translation $[13,15]$. Under the new naming rules, the new miRNA is no longer represented by miRNA and miRNA*, but named miRNA -3p and miRNA $-5 p$ based on the position of the mature miRNA in the precursor [16]. miRNA $-3 p$ is close to the 3 'end of the precursor, while miRNA -5p is close to the 5 'end of the precursor [17].

A large number of miRNAs are evolutionary conserved among diverse species within the plant kingdom and these miRNAs usually have conserved regulation mechanism, such as the expression of a class III homeodomain-leucine zipper (HD-Zip III) protein and miR166, APETALA2 and miR172 [18]. Previous studies have showed that there are several miRNAs with species-specificity in each plant and these miRNAs may participate in morphogenesis of organs and plant defense response. However, most of the species-specific miRNAs remained unidentified in many plant species because of their lower levels relative to conserved miRNAs $[18,19]$. In recent decades, with the advent of high-throughput sequencing technology, both species-specific miRNAs have been identified in diverse plant species [20-23]. Furthermore, the expression of miRNAs also shows specificity of period and tissue, and it is possible to identify new miRNAs from different tissues or organs at different developmental stages.

Pseudostellaria heterophylla is a kind of Chinese medicinal material widely distributed in China and has important economic value. Its tuberous root was used and called Pseudostellariae Radix, which contains polysaccharides, saponins, phospholipids, cyclopeptides, fatty acids, and volatile components [24]. Pseudostellariae Radix was commonly used for protection of myocardial, enhancement of immunity, anti-oxidation, hypoglycemia and anti-fatigue [25]. Although there are a lot of studies on the chemical composition and pharmacological action of $P$. heterophylla in recent years [26-27], the exploration about the regulation mechanism of plant growth and development and continuous cropping obstacles is still needed. In previous studies, a transcriptome database was constructed from different organs and some endophytes were also found [28]. In this study, we report a population of $P$. heterophylla miRNAs from vegetative organs (leaf, stem,

Page $2 / 21$ 
xylem and bark of tuberous root). The conserved and species-specific miRNAs were both identified by searching the newly constructed transcriptome of $P$. heterophylla. The correlation in the expression pattern of miRNAs and their target mRNA was analyzed combined with the transcriptome. Gene Ontology (GO) and the Kyoto Encyclopedia of Genes and Genomes (KEGG) were used for exploring the functions of the predicted potential miRNA targets. The target genes of conserved and species-specific miRNAs were validated through 5'RLM-RACE. The reported mRNA of 5 endophytes were downloaded from GenBank and used for searching the targets gene of miRNA in $P$. heterophylla. These results may help to explain the function of miRNA in morphogenesis of organs and defense of endophytes in $P$. heterophylla, and provide theoretical basis for breeding and genetic improvement of $P$. heterophylla.

\section{Materials And Methods}

\section{Plant material and Total RNA isolation}

P. heterophylla plants (Shitai NO.1) were selected by our group and certified as a national variety by Guizhou Crop Variety Approval Committee in 2016 (Accession number: 2016002). It has become a very popular variety in Guizhou Province because of its excellent quality and high yield. Plants were grown in experimental field of Shibing County, Guizhou Province, China. The leaves, stem and tuberous root (cortex and xylem) of $P$. heterophylla were collected separately from three randomly selected individuals. After cleaning, all samples were immediately frozen in liquid nitrogen. Transzol Plant RNA Extraction Kit (TaKaRa, Japan) was used for extraction of total RNA accprding to the manufacturer's instructions. Traces of remaining DNA were removed by using DNase I (Takara, Tokyo, Japan).

\section{cDNA library preparation and transcriptome sequencing}

For a better understanding of miRNA and mRNA networks in $P$. heterophylla, the cDNA library of these tissues was also constructed. The total RNA of four tissues were purified by using Oligo(dT) magnetic beads, respectively. The mRNA was disrupted into small fragments and using for second-strand CDNA synthesis. These CDNA fragments were ligated with the lllumina paired-end sequencing adaptors and sequenced by using Illumina Hiseq4000 platform. The expression level of each gene in different organs was normalized by using the reads per kb per million reads (RPKM) and the RPKM of each unigene refer to the sum of RPKM from all isoforms of the same gene.

\section{Small RNA library construction}

Small RNA libraries for each sample were prepared independently using Truseq TM Small RNA sample prep Kit (Illumina, U.S.A). Total RNAs of $P$. heterophylla were firstly size-fractioned on $15 \%$ denaturing polyacrylamide gel and small RNAs (18-35nt) fraction was collected. Next, T4 RNA ligase was used to ligate the small RNAs and adapters (5'and3'). Small RNAs with adapters were reverse-transcribed using SuperScript II Reverse Transcriptase (Invitrogen, U.S.A) and PCR amplified with PCR. The amplification products were excised from the Novex 6\% TBE-Urea Gel. The cDNA samples were sequenced using Illumina Hi-seq2000 at Shanghai Majorbio Bio-pharmTechnology Co.,Ltd. (Shanghai,China). To obtain the clean reads, reads with low-quality reads, adaptor sequences and the reads shorter than 18nt and longer than 30nt were removed.

\section{Identification of conserved and novel miRNAs}

These high-quality sRNA sequences were mapped to the mRNA transcriptome database. These perfectly mapped sRNAs were used for further analysis, while rRNA, tRNA, small nuclear (snRNA), and small nucleolar RNA (snoRNA) were removed. Remaining sRNAs were aligned with the mature miRNA or miRNA precursor from all plant species in the miRBase Sequence Database (http://www.mirbase.org/, release 18.0, November 2011) with allowance of two mismatches. These perfectly matched sRNAs were collected and considered to be conserved miRNAs. Potentially novel miRNAs were predicted using MIREAP (http://sourceforge.net/projects/mireap/), and the secondary structure of the sRNA precursor were calculated by using the RNAfold web server [31] (http://rna.tbi.univie.ac.at/cgi-bin/RNAfold.cgi) with default parameters. Those sRNAs fitting all the published criteria and with no homologous sequence in the public databases was considered to be a putative novel miRNA precursor. The expression level of miRNAs was normalized to one million by total clean reads of miRNAs in each sample sequence. For the novel miRNAs, the names from Phn-miR1 to Phn -miR303 were given.

\section{Prediction of miRNA targets and $\mathrm{GO}$ analysis}

The predication of miRNA targets were performed by using the Target Finder (https://github.com/carringtonlab/TargetFinder) against the transcriptome sequence data of $P$. heterophylla in previous study. Maximum expectation and complementarity scoring were used for evaluating the miRNA-target mRNA interactions according to the method described by Allen et al [19].

For a better understanding the function of the target genes and their corresponding metabolic network regulated by these miRNAs, Gene Ontology (GO) and the Kyoto Encyclopedia of Genes and Genomes (KEGG) were used. 
To analysis the regulation of miRNAs and their targets genes, the expression level of Spearman correlation coefficients between the miRNAs and their targets genes were performed.

\section{Target validation of conserved miRNAs by RLM-RACE}

The cleavage site of predicted targets was determined by using a 5' RLM-RACE (Takara, Tokyo, Japan) according to the instructions of the manufacturer. Briefly, RNA adapter was ligated to the mRNA using the T4 RNA ligase and reverse transcribed to cDNA. Gene-specific reverse primers and 5'adapter primers were used to amplify the cleaved transcripts. The primers used to amplify cleavage products of miRNA target genes in P. heterophylla are listed in Additional file 16. The amplifying fragments were cloned to pMD19-T vectors (Takara, Tokyo, Japan) and sequenced by M13 primers.

\section{Results}

\section{An overview of high-throughput sequencing data sets}

To identify the miRNA in P. heterophylla, a small RNA library from four organs (leaves, stem, tuberous root cortex and xylem) was constructed and sequenced independently. A total of 180 million raw reads were obtained from these 12 libraries. These raw data were filtered through several criteria to identify conserved and specific miRNAs.

The length distribution of the small RNAs was shown in Fig 1. The majority of reads were 21 to 24 nt in length. The 24 -nt RNA is the most abundant class in the sRNA library and comprised $35.7 \%$ of root bark, $32.7 \%$ of root xylem, $31.5 \%$ of leaf and $31.8 \%$ stem. Similar results were reported in potato, Arabidopsis thaliana, tomato, pepper, cucumber [29], but in contrast to grapevine, wheat [30].

\section{The transcriptome of $P$. heterophylla}

We obtain $603,398,664$ raw reads and 598, 156, 596clean reads from four organs with the base average error rate below $0.02 \%$. Trinity program was used for de novo assembling of clean data. 138, 888 unigenes and 207, 390 transcripts were obtained. The length of unigenes ranged from 201 to 14, 793 bp and the N50 length was 1, 862. 71, 215 unigenes (51.28\%) and 121, 508 Transcripts (58.59\%) were annotated based on the public databases. These sequences were used for identifying the miRNAs.

\section{Identification of known miRNAs in P. heterophylla}

In order to identify the conserved miRNAs in P. heterophylla, the small RNA were submitted to the miRBase 21 (http://www.mirbase.org/). Sequence with high identify to other species were considered as known miRNA. A total of 163 miRNAs belonging to 66 families were identified (Additional file 1). Among these families, the miRNA169 has 13 members distinguished based on nucleotide differences and followed by miRNA166 (12 members each); miRNA156 (11 members each); miRNA395 (9 members each); miRNA160 (8 members each) and miRNA399 (8 members each). The remaining miRNA families comprised 1 to 6 members each (Fig 2).

Of the 66 conserved miRNA families, miRNA396, miRNA159, miRNA319 and miRNA166 showed high abundance with total TPM >100 000 (Additional file 1). The highest read abundance (7 202956 normalized reads) was detected for miR396. However, 37 miRNA families with total TPM less than 10 were found, such as miR1871, miR829, miR828 and miR2873. These results suggested variations in expression levels of miRNAs and their highly conserved function.

\section{Identification of novel candidate miRNAs in $P$. heterophylla}

The non-conserved small RNA reads were mapped to unigenes in transcriptome of $P$. heterophylla and a total of 303 novel miRNA candidates were identified (Additional file 2). A standard stem-loop hairpin secondary structure (SS) was found in all miRNA precursors. The secondary structure of precursors of novel miRNAs were predicted by using Mfold web server [31] and a few were showed in Figure 3. These miRNA precursors had folding free energies ranging from -48 to $-18 \mathrm{kcal} / \mathrm{mol}$ (average, $-27.28 \mathrm{kcal} / \mathrm{mol}$ ). The length of predicted precursor of these novel miRNAs were 58-101 nt. The MFEl values of all miRNAs were above 0.85 and these sequences were considered as miRNA[32]. Of the 303 novel miRNA, 4 novel miRNAs had high expression level with TPM $>1000$. The highest read abundance (2 662 normalized reads) was detected for Phn-m0190. 73 novel miRNA with total TPM less than 10 were found, such as Phn-m0032, Phn-m0045, Phn-m0050, Phn-m0058 and Phn-m0076.

\section{Prediction of miRNA target genes}


To explore the biological functions of the identified miRNAs, Target Finder program were used for searching the complimentary mRNA sequences from transcriptome sequence data of $P$. heterophylla above. Among the conserved targets, numbers of transcripts coding for transcription factors were identified (Additional file 3), such as GAMYB-like (targeted by miR159), APETALA2 and bHLH (target of miR172), PHAVOLUTA-like HD-ZIPIII protein (target of miR166), nuclear transcription factors - YA3, YA5, Zinc finger CCCH domain-containing protein and WRKY (target of miR169), NAC domain containing protein (targeted by miR164), Squamosa promoter-binding protein and MADS (regulated by miR156), TCP 4(targeted by miR319), Auxin response factor (regulated by miR160). This result suggested that they may participate in post-transcriptional regulation and transcriptional networks.

For novel miRNAs, 305 potential target genes involving with protein kinase were identified (Additional file 4), such as serine/threonineprotein kinase-like protein CCR4 (TRINITY_DN90887_c1_g2), L-type lectin-domain containing receptor kinase S.7

(TRINITY_DN87850_c4_g1), LRR receptor-like serine/threonine-protein kinase (TRINITY_DN93055_c2_g2 and TRINITY_DN93285_c2_g1) and plastidial pyruvate kinase 4 (TRINITY_DN88253_c0_g1). 162 potential target unigenes coding transcription factors were also observed, including MYB (44 unigenes), bHLH (34 unigenes), WRKY (15 unigenes) and MADS (12 unigenes). In addition, 34 potential target genes were founded to be involving with the synthesis and biotransformation of NADPH. These results indicated that novel miRNAs in $P$. heterophylla may participate in several diverse physiological and metabolic processes, including the regulation of plant metabolism, transport, cell growth and maintenance, and stress responses.

In addition, multiple potential regulation patterns were discovered in the same unigenes (Additional file 5). 8 binding site of Phn-m051-5p and Phn-m052-5p were identified in the mRNA sequence of ubiquitin-conjugating enzyme E2 24 (TRINITY_DN90017_c1_g1).

transmembrane protein 97 (TRINITY_DN86988_c0_g5) have 11 potential regulation patterns target by miRNA399 families, Phn-m0052-3p, Phn-m0051-5p, Phn-m0052-5p and Phn-m0220-5p. Three HD-ZIPIII protein, ATHB8 (TRINITY_DN91558_c0_g5), ATHB15

(TRINITY_DN91558_C0_g3) and REVOLUTA (TRINITY_DN91558_c0_g7), have 9 potential regulation patterns target by miRNA166 families, Phn-m0140-3p and Phn-m0141-3p. Furthermore, several unigenes were only targeted by novel miRNA, such as multicopper oxidase LPR2 (TRINITY_DN90101_C3_g6), L-ascorbate oxidase homolog (TRINITY_DN92548_C7_g1), ribosomal RNA-processing protein 7 homolog A (TRINITY_DN90150_c1_g2) and pyrrolidone-carboxylate peptidase (TRINITY_DN87378_c4_g1). To summarize, the novel miRNA in $P$. heterophylla show an extreme important role in regulation of growth and development.

\section{GO and KEGG analysis}

GO analysis was performed to explore the potential regulation of predicted target transcripts of miRNAs in P. heterophylla. Based on their functional annotations, the target genes were into 122 categories (Additional file 6). In particular, 6 categories have higher enrichment, such as protein kinase activity, protein serine/threonine kinase activity, phosphotransferase activity, alcohol group as acceptor, calcium ion binding, kinase activity and response to acid chemical (Fig 4 ). KEGG pathway enrichment analysis showed that the target genes of the miRNAs were mainly involved in 8 pathways ( $P \leq 0.05$ ): Plant-pathogen interaction, MAPK signaling pathway-plant, plant hormone signal transduction, ubiquitin mediated proteolysis, phosphatidylinositol signaling system, phenylpropanoid biosynthesis, inositol phosphate metabolism and diterpenoid biosynthesis (Additional file 7 and Fig 5). These results suggested that the miRNAs in $P$. heterophylla may play an important role in regulating cell signal transduction.

\section{Role of miRNAs in ground and underground parts}

For a better understanding of miRNAs in morphogenesis of organs, some tissue-special miRNAs were analyzed by combining with the transcriptome (Additional file 8). 6 conserved miRNAs was found to be most abundant in ground parts and could rarely be detected in underground parts, including 2 member of miR172 family (miR172e-5p and miR172h-5p), miR156a-3p, miR167g-3p, miR397b and miR398a-3p. 9 novel miRNA showed special expression in ground parts, including Phn-m021-5p, Phn-m0028-3p, Phn-m0084-3p, Phn-m0121-5p, Phn-m0147-3p, Phn-m0170-3p, Phn-m0173-5p, Phn-m0292-5p and Phn-m0246-5p (Fig 6). 115 potential target genes were identified for 18 miRNAs (Additional file 9), including 33 coding unigenes and 82 non-coding unigenes. 16 unigenes target by Phn-m0170-3p and 6 genes target by Phn-m0173-5p were identified. CRK7 (TRINITY_DN92623_c1_g1), PUB6 (TRINITY_DN88470_c4_g3) and BBX19 (TRINITY_DN86966_c1_g1) showed significantly negative correlation with the expression of Phn-m0173-5p. SODC (TRINITY_DN88258_c4_g4) was negatively regulated by miR398a-3p.

12 conserved miRNAs showed abundant expression in underground parts (root bark and xylem), while be restricted to ground parts (stem and leaf), including 3 member of miR164 family (miR164b, miR164c and miR164e), 4 member of miR169 family (miR169a, miR169a-3p, miR169j and miR169y), 2 member of miR1863 family (miR1863a and miR1863b), miR156b-3p, miR172c and miR6470. 13 novel miRNAs also high expression in underground parts (root bark and xylem), including Phn-m0008-3p, Phn-m0274-3p, Phn-m0103-5p, Phn-m0080-5p, Phn-m0114-5p, Phn-m0046-5p, Phn-m0238-3p, Phn-m0151-5p, Phn-m0167-5p, Phn-m0286-5p, Phn-m0035-5p, Phn-m0046-5p and Phn-m0113-3p. 107 potential target genes were identified for 18 miRNAs, including 37 coding mRNA and non-coding

Page 5/21 
mRNA. It is founded that the expression profiles of two conserved miRNA, miRNA169a and miRNA169a, and Phn-m0046-5p were negatively correlated with NF-YA5 (TRINITY_DN85133_c0_g1) and PP2C08 (TRINITY_DN86665_c2_g1) respectively, which is in accordance with the gene-silencing function. Additionally, the expression of several non-coding unigenes showed significantly positive and negative correlation with these miRNAs.

\section{Role of miRNAs in development of leaf}

Interestingly, lot of miRNAs showed special expression profile in a certain organs. 9 conserved miRNAs and 2 novel miRNAs and could rarely be detected in leaf (Fig 7 and Additional file 10), including 5 member of miR319 family (miR319a, miR319a-3p.2-3p, miR319b-5p, miR319d5p and miR319i), miR164f, miR171d, miR390b, miR394a-5p, Phn-m0151-5p and Phn-m0113-3p. 36 potential target genes were identified for 11 novel miRNAs, including 13 coding mRNA and 23 non-coding mRNA. In particular, the TCP (TRINITY_DN90304_c1_g3, TRINITY_DN89893_c1_g2 and TRINITY_DN89893_c1_g1) show significantly negative correlation with 3 miRNA319 families, miR319a, miR319a-3p.2-3p and miR319i. Interestingly, 3 non-coding mRNA have extremely positive correlation with the miRNA319 family. These results suggested that miR156a, miR159 family and miR319 family play an important role in development of leaf.

However, 19 conserved miRNAs and 2 novel miRNAs show abundant expression in leaf, including 3 members of miR156a (miR156a, miR156a-3p and miR156a-5p), 3 members of miR159 (miR159a, miR159d and miR159c), 2 members of miR167 (miR167h-5p and miR167d), 2 members of miR168(miR168b-5p and miR168a), 4 members of miR395 (miR395a, miR395c, miR395h and miR395k), 2 members of miR396 (miR396a-3p and miR396b-3p), miR171c-5p, miR172b, miR2111f, Phn-m0026-3p and Phn-m0222-5p. 88 potential target genes were identified for 21 novel miRNAs, including 29 coding mRNA and 59 non-coding mRNA. miR156a targeted 10 unigenes and GAMYB (TRINITY_DN91612_c2_g3 and TRINITY_DN89557_c1_g5) may regulated by miR159 families. Though none of miRNA showed negative correlation with the target unigenes, some unigenes have positive correlation with miRNAs, such as GAMYB (target by miR159a, miR159c and miR159d), EAF6 (target by miRNA156a) and receptor-like protein kinase At2g23200 (target by miR172b).

\section{Role of miRNAs in development of stem}

3 conserved miRNAs and 12 novel miRNAs have abundance transcripts in stem (Fig 8 and Additional file 11), including miR828b-5p, miR169d, miR156e, Phn-m0140-3p, Phn-m0208-5p, Phn-m0098-3p, Phn-m0282-5p, Phn-m0082-3p, Phn-m0291-5p, Phn-m0077-5p, Phn-m0252-3p, Phn-m0038-5p, Phn-m0295-3p, Phn-m0044-5p, Phn-m0024-3p. 239 potential target genes were identified for 15 miRNAs, including 75 coding mRNA and 164 non-coding mRNA. 36 coding mRNA target by Phn-m0082-3p, 10 coding mRNA target by Phnm0077-5p and 7 coding mRNA target by Phn-m0024-3p. The expression of snakin-1 (TRINITY_DN90759_c4_g6) and PUB3 (TRINITY_DN90343_c1_g6) has negative correlation with Phn-m0082-3p and Phn-m0024-3p, respectively. However, miR156I-5p and Phn-m0224-3p showed low expression in stem whereas relatively high expression was observed in underground parts and leaf. The expression abundance of miR156I-5p showed negative correlation with SPL5 (TRINITY_DN90116_c0_g1).

\section{Role of miRNAs in development of tuberous root}

To explore the function of miRNAs in development of tuberous root, tissue-special miRNAs in xylem and bark were identified. 12 novel miRNAs were especially up-regulated in root xylem (Fig 9 and Additional file 12), including Phn-m0262-3p, Phn-m0217-3p, Phn-m0249-3p, Phn-m0261-5p, Phn-m0101-5p, Phn-m0198-3p, Phn-m0279-3p, Phn-m0274-5p, Phn-m0100-5p, Phn-m0184-3p, Phn-m0092-5p, Phn-m0214-5p. 96 potential target genes were identified for 12 novel miRNAs, including 36 coding mRNA and 60 noncoding mRNA. Though 24 coding unigenes was targeted by Phn-m0101-5p and 6 coding unigenes was targeted by Phn-m0279-3p, none of miRNA showed negative correlation with the target unigenes.

However, 16 conserved miRNAs and 5 novel miRNAs specially showed low expression in root xylem, including 7 member of miR 166 family (miR166a-3p, miR166b-5p, miR166g-5p, miR166i-3p, miR166k, miR166k-3p and miR166n), 2 member of miR160 family (miR160b-3p and miR160d-5p), 2 member of miR171 family (miR171c-3p and miR171e-3p), 2 member of miR399 family (miR399a and miR399h), miR159b-5p, miR165a-3p, miR393a-5p, Phn-m0225-3p, Phn-m0162-5p, Phn-m0086-5p, Phn-m0042-5p and Phn-m0022-5p. 77 potential target genes were identified for 21 miRNAs, including 38 coding mRNA and 39 non-coding mRNA. Three HD-ZIP III proteins were extreme negatively regulated by miR166 family. It is seem that SCL6, BIR2, ARFR and WDL5, were negatively regulated by miR171 family, Phn-m0162-5p, miR160d-5p and miR166g-5p in xylem of tuberous root, respectively. These results suggested that these miRNAs play an important role in in development of tuberous root.

In addition, 7 conserved miRNAs were especially up-regulated in root bark (Fig 10 and Additional file 13), including 3 member of miR160 family (miR160f-5p, miR160g and miR160h), 2 member of miR390 family (miR390-3p and miR390d-3p), miR169w, miR171a. However, none of miRNA showed negative correlation with its target gene.

Page 6/21 
Hormone plays a curial role in the morphogenesis of plant organs. In this study, we analyzed regulation function of miRNA on hormone signal transduction by searching the target genes of miRNAs. The results showed that a large number of genes in most of the hormone signal transduction pathways were targeted by identified miRNAs in $P$. heterophylla, mainly involving with signal transduction of Auxin, Zeatin, Abscisic acid and Jasmonic acid (Fig 11 andAdditional file 14). Five genes in Auxin signal transduction pathway, TIR1, Aux/IAA and ARF, may heavily be regulated by miRNAs in $P$. heterophylla. SAUR is a multiple family and targeted by 10 miRNAs, including 2 known miRNAs and 8 novel miRNAs. In CTK signaling transduction, there were several binding sites of miRNAs in the sequences of AHP, B-ARR and A-ARR. Interestingly, PYL, PP2C and SnRK2 in ABA signal transduction pathway may strongly be regulated by miRNAs, mainly composed of novel miRNAs. We also founded that few novel miRNAs may regulate the expression of COI1 and JAZ in jasmonic acid signaling transduction pathway. In addition, gibberellin receptor GID2 in gibberellin signal transduction was targeted by Phn-m0174-5p. BSK in Brassinosteroid signaling transduction pathway was targeted by 3 known miRNAs and 5 novel miRNAs. A few miRNAs were identified involving with the ethylene signal transduction, such as MPK6 (targeted by Phn-m0087-5p, Phn-m0154-3p and Phn-m0281-5p), EBF1/2 (targeted by Phn-m0033-5p), and EIN3 (targeted by mir166e-5p and Phn-m0122-3p).

\section{Role of miRNAs in interaction between plant and entophytes}

For a better understanding of the role of miRNAs in interaction between plant and endophytes, mRNA sequences of 4 microorganisms (Fusarium oxysporum, Leptosphaeria maculans, Microbotryum violaceum, Rhodosporidium toruloides) were downloaded from Genbank and used for searching the target genes of miRNAs of $P$. heterophylla (Fig 12 andAdditional file 15). 39 genes of from F. oxysporum were targeted by 30 miRNAs of $P$. heterophylla, including 15 known miRNAs and 15 novel miRNAs. 6 genes were targeted by miR156h, followed

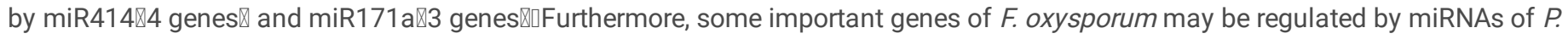
heterophylla, such as pectin lyase $F$ \target by Phn-m0104-3p囚, 30S ribosomal protein S17-likeprotein (miR171e-3p) and myo-inositol

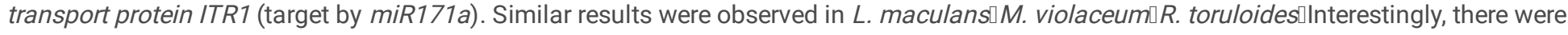
37, 43, 27 genes in $L$. maculans $\square$. violaceum $R$. toruloides targeted by miR414, respectively. This result suggested that miR414 may play an important role in interaction between $P$. heterophylla and endophytes.

\section{miRNA-induced cleavage of predicted targets}

Cleavage site of predicted targets of a conserved miRNA and a novel miRNA in

1. heterophylla were further verified by 5' RLM-RACE (Fig 13). The predicted targets of miR166k and Phn-m0121-5p were HD-ZIP REVOLUTA and delta(24)-sterol reductase, respectively. All the fragment of 5'RLM-RACE were analysed on agarose gel, purified, cloned and sequenced. The cleavage site was found to be between $2-3$ bases, from the 5 ' end of the miRNA, for HD-ZIP REVOLUTA (TRINITY_DN91558_c0_g3). However, Delta (24)-sterol reductase (TRINITY_DN86257_c0_g3) was found to be cleaved between 5-6 from the 5 'end of the miRNA binding site.

\section{Discussion}

In earlier studies, numbers of miRNAs were identified from various plants through computational and direct cloning approaches [19-23]. However, plenty of miRNAs show specifical expression similar to the coding genes and were identified from different tissues of certain period. For example, about one hundred of miRNAs were from each organs of each plant, but 89 conserved miRNAs and 3631 novel miRNA were discovered from root, stem, leaf and tuber developmental stages of potato. In this study, 163 conserved miRNAs and 303 level miRNAs were identified and some of them showed specifically up or down regulated expression in leaf, stem, xylem and bark of tuberous root. In addition, $22 \mathrm{nt}$ and $24 \mathrm{nt}$ of sRNA have higher abundance of transcripts in xylem of root tuber, which suggested that these sRNA play an important role in the growth and development of tuberous root.

In fact, morphogenesis of organs depends on expression pattern of genes in plants induced by different environment factors, such as soil, water, temperature, sugar transport and microorganisms. Previous studies had indicated that the expression of genes showed extreme difference between ground parts (leaves and stems) and underground parts (bark and xylem of tuberous root), while transcript abundance of few genes have significant different between bark and xylem of tuberous root [28]. This result suggested that there were some similar regulatory mechanisms in several organs of ground parts or underground parts. In this study, the expression patterns of miRNA in different tissues were analyzed and used to reveal its role in organ morphogenesis. Two members of miRNA172 family and 4 conserved miRNAs in the aboveground part were significantly up-regulated. A few members of miRNA164 gene family, miRNA169 gene family and miRNA1863 gene family were significantly up-regulated in the underground parts. It has been reported that miRNA172 might be associated with curl symptoms of leaf, and its target gene APETALA2-Like may affect the flowering time of Arabidopsis [18]. miRNA164 can reduce the 
formation of lateral roots by down-regulating the expression level of $N a C 7[33]$. miRNA169 can affect the number and size of certain cells in root meristem and the initiation of lateral roots [34]. miRNA169 family also participated in the response of roots to environmental stresses , such as drought and salt stress [35]. Interestingly, expression level of miRNA1863 showed no significant correlation with the target genes M810, SHGR9 and TPR1, but TPR1 together with MYB44 may regulate the expression of PP2C gene. These results suggesting that these miRNAs may play an important role in the growth and development of tuberous roots.

Further analysis showed that miRNA319 gene family was specifically down regulated in leaves, and may affect leaf morphogenesis by regulating TCP4 expression, hormone synthesis and signal transduction pathway[35]. GAMYB were also targeted by miR319i and miR159 family, which suggested their important function in the growth and development of leaves [37]. However, we found that some members of miR395 family, miR167 family and Phn-m0170-3p were significantly up-regulated in the leaves and phn-m0170-3p may be involved in the regulation of 16 coding genes. miR156e and miR156I were specifically up-regulated in stem, may affect the expression of the target gene SPL, prolong the time of vegetative growth, delay the flowering, regulate the plant architecture, and affect the formation of anthocyanins in the stem of $P$. heterophylla[38]. It is worth noting that Phn-m0082-3p is specifically expressed in the stem and may regulate the expression of 36 coding genes, and its function needs further study.

As a storage organ, tuberous root can not only provide an important mechanism for overwintering of plants, but also provide nutrition for plant growth in the next year. At present, many kinds of tuberous root have been used for food or development of medicine all over the world [39]. Therefore, the development regulation of tuberous root has attracted more and more attention. In fact, the formation of tuberous root mainly depends on the formation of secondary xylem from phloem and expansion of parenchyma cells [40]. Therefore, the development regulation of xylem plays a crucial role in the formation of tuberous root. In this study, several members of miR166, miR171, mirna160, miR399 were significantly down regulated in the xylem of tuberous roots of $P$. heterophylla and which suggested an important role. The down-regulated expression of miR171 may affect the growth and development of tuberous roots in $P$. heterophylla by regulating three target genes of the scar like family, namely SCL6-II, SCL6-III and SCL6-IV [41-42], controlling the radial growth of roots [43] and hormone signal [44]. Overexpression of miR166 can reduce the number of and lateral roots and symbiotic nodules and induce the ectopic development of vascular bundles in these transgenic roots [45]. This result indicated that down-regulated expression of miR166 in the tuberous root of $P$. heterophylla can promote the up-regulated expression of homeodomain leucine zipper (HD-Zip III) genes, affect the development process of leaf polarity establishment and vascular tissue differentiation, and which suggested that miR166 family and its target gene (HD-Zip III) may also play an important role in development of tuberous root. Some members of ARF family were targeted by miR160, such as ARF 10, ARF 16 and ARF 17. miR160 has showed extreme importance in differentiation of root cap cells, morphogenesis of embryo and leaf, development of flower[34-46], and mainly regulated the expression of its targets genes (AFR). 12 novel miRNAs were significantly upregulated in the xylem of tuberous root and 24 unigenes were targeted by Phn-m0101-5p, which suggested its important role in the development of root xylem. In addition, many members of miR390 were specifically up-regulated in the bark of tuberous root, which may regulate the development of tuberous root by responding to auxin.

miRNA not only directly regulates the development of plants through its target genes, but also indirectly affects the morphogenesis of organs by regulating the expression of genes involving in hormone signal transduction. Previous studies have shown that miRNAs were involved in the signal transduction of auxin and ABA [47]. miR393 can regulate the ubiquitination of Aux/IAA protein by affecting the expression of TIR1 [48]. Overexpression of miR393 also slowed the plant growth by reducing level of auxin [49]. miR167 can regulate the expression of $\mathrm{GH} 3$ and affect the accumulation of indole-3-acetic acid though its target genes ARF6 and ARF 8 [50]. In addition, miR160 and miR167 may be a cross regulator of ABA and auxin signal transduction regulation [51]. In this study, we found that 317 kinase related genes were targeted by miRNA (supplementary materials), and which were mainly enriched in the pathway of plant-pathogen interaction, MAPK signaling pathway-plant, ubiquitin mediated proteolysis and plant hormone signal transduction. These results suggested that the miRNA of $P$. heterophylla may be involved in the response to biological and abiotic stress in plants.

Further analysis showed that the miRNA of $P$. heterophylla not only had a strong regulatory role in the signal transduction pathway of auxin and abscisic acid, but also participated in the signal transduction of CTK, gibberellin, ethylene and jasmonate. Previous studies showed that the contents of ABA, CTK (ZR and DHZR) and JA increased gradually with the development of tuberous roots, and which involved in the regulation of cell proliferation, cell elongation, cell expansion [52], formation of microtubule microfilaments [53] and accumulation of assimilates in tuberous roots [54]. These results suggested that the miRNA of $P$. heterophylla may affect the development of tuberous roots by regulating its target gene in pathway of hormone signal transduction.

Many researches have proved that there are complex interaction mechanisms between plants and pathogenic microorganisms, and plants have evolved perfect defense mechanisms, including miRNA system. miR393 was firstly discovered and negatively regulated by the pathogen[55]. After infected by 4 tobacco mosaic viruses, the expression of miRNA168 was significantly up-regulated [56]. A variety of new miRNAs could be detected in plants infected by rice stripe virus [57]. In our previous studies, five kinds of endophytes were identified in $P$. 
heterophylla by transcriptome sequencing, in which M. violaceum, L. maculans, and $P$. fluorescens are typical harmful bacteria [28]. $F$. oxysporum is closely related to the continuous cropping obstacle of $P$. heterophylla. In this study, a large number in mRNA of four kinds of endophytes were targeted by miRNAs of $P$. heterophylla, and there were several genes targeted by miR414, while none of unigenes in $P$. heterophylla were targeted by miR414. These results suggested that miR414 may provide a new way to overcome the disease resistance and continuous cropping obstacles of plants. However, miR414 showed extreme low expression in the tissue of $P$. heterophylla and it is necessary to clarify its function in the defense of microorganisms.

Furthermore, a large number of long-chain non coding RNAs (IncRNA) were targeted by miRNAs in $P$. heterophylla. There was a significant negative correlation between the expression level of some IncRNAs and miRNA. It has been proved that IncRNA plays an important role in plant growth and development, fertility, biotic and abiotic stress response and other biological processes [58]. miRNAs can not only directly act on IncRNA, but also interact with the overlapping of miRNA regulatory network or the special relationship between the two positions [59]. Therefore, miRNA can not only directly regulate the expression of coding genes, but also indirectly affect the expression of coding genes by influencing the expression of long-chain non coding RNA (IncRNA) [60]. For a better understanding of physiological function of miRNA in $P$. heterophylla, we must understand the regulation function of IncRNA targeted by miRNA.

\section{Declarations}

\section{Acknowledgements}

Not applicable.

\section{Author Contributions}

JL, CMW, TZ and XQL designed research. JL and HJJ performed research. XQL and HJJ analyzed data. CMW, JL and HJJ draft the manuscript. All authors read and approved the final manuscript.

\section{Funding}

This work was supported by grants from the National Key R\&D Program of China (NO. 2019YFC1712500), National Natural Science Foundation of China (NO. 81860667), the Science and Technology Department of Guizhou Province (QKHHBZ[2020]3003), First-class Discipline Construction Projects of Guizhou Province of China (GNYL(2017)008), the Science and Technology Department of Guizhou Province (QKHJC[2018]1010).

\section{Ethics approval and consent to participate}

All authors declare that the experiments complied with current laws in which they were performed.

\section{Materials statement}

All authors comply with the IUCN Policy Statement on Research Involving Species at Risk of Extinction and the Convention on the Trade in Endangered Species of Wild Fauna and Flora.

\section{Availability of data and materials}

The datasets used and/or analyzed during the current study available from the corresponding author on reasonable request.

\section{Consent for publication}

Not applicable.

\section{Competing interests}

The authors declare that they have no competing interests.

\section{Author details}

Guizhou University of Traditional Chinese Medicine, Guiyang, 550025, China

\section{References}


1. Chen X. Small RNAs and their roles in plant development. Annu Rev Cell Dev Biol. 2009; 25(1): 21-44.

2. Li YF, Zheng Y, Addo-Quaye C, Zhang L, Saini A, Jagadeeswaran G, Axtell MJ, Zhang,W., Sunkar,R. Transcriptome-wide identification of microRNA targets in rice. Plant J. 2010; 62(5): 742-59.

3. Lee RC, Feinbaum RL, Ambros V. The elegans heterochronic gene lin-4 encodes small RNAs with antisense complementarity to lin-14. Cell. 1993; 75(5): 843-54.

4. Xie F, Frazier TP, Zhang B. Identification and characterization of microRNAs and their targets in the bioenergy plant switchgrass (Panicum virgatum). Planta. 2010; 232(2): 417-34.

5. Chuck G, Candela H, Hake S. Big impacts by small RNAs in plant development. Curr Opin Plant Biol. 2009; 12(1): 81-86.

6. Hwang EW, Shin SJ, Yu BK, Byun MO, Kwon HB. miR171 Family members are involved in drought response in Solanum tuberosum[J]. J Plant Biol. 2011; 54(1):43-48.

7. Dai ZY, Tan J, Zhou C, Yang XF, Yang F, Zhang SJ, Sun SC, Miao XX, Shi ZY. The OsmiR396-OsGRF8-OsF3H-flavonoid pathway mediates resistance to the brown planthopper in rice (Oryza sativa). Plant Biotechnol J. 2019; 17(8): 1657-1669.

8. Du P, Wu JG, Zhang JY, Zhao SQ, Zheng H, Gao G, Wei LP, Li Y. Viral infection induces expression of novel phased microRNAs from conserved cellular microRNA precursors. PLoS Pathog. 2011; 7(8): e1002176.

9. Lee Y, Kim M, Han J, Yeom KH, Lee S, Baek SH, Kim VN. MicroRNA genes are transcribed by RNA polymerase II. EMBO J. 2004; 23(20): 4051-4060.

10. Li JJ, Yang ZY, Yu B, Liu J, Chen XM. Methylation protects miRNAs and siRNAs from a 3'-end uridylation activity in Arabidopsis. Curr Biol. 2005; 15(16): 1501-1507.

11. Park MY, Wu G, Gonzalez-Sulser A, Vaucheret H, Poethig RS. Nuclear processing and export of microRNAs in Arabidopsis. Proc Natl Acad Sci U S A. 2005; 102(10): 3691-3696.

12. Kurihara Y, Watanabe Y. Arabidopsis micro-RNA biogenesis through Dicer-like 1 protein functions. Proc Natl Acad Sci U S A. 2004; 101(34): 12753-12758.

13. Vazquez F, Gasciolli V, Crété $P$, Vaucheret $H$. The nuclear dsRNA binding protein HYL1 is required for microRNA accumulation and plant development, but not posttranscriptional transgene silencing. Curr Biol. 2004; 14(4): 346-351.

14. Jones-Rhoades MW, Bartel DP. Computational identification of plant microRNAs and their targets, including a stress-induced miRNA. Mol Cell. 2004; 14(6): 787-799.

15. Baumberger N, Baulcombe DC. Arabidopsis ARGONAUTE1 is an RNA Slicer that selectively recruits microRNAs and short interfering RNAs. Proc Natl Acad Sci U S A. 2005; 102(33): 11928-11933.

16. Kozomara A, Griffiths-Jones S. miRBase: annotating high confidence microRNAs using deep sequencing data. Nucleic Acids Res. 2014; Database issue): D68-73.

17. Seitz H, Ghildiyal M, Zamore PD. Argonaute loading improves the 5' precision of both MicroRNAs and their miRNA* strands in flies. Curr Biol. 2008; 18(2): 147-151.

18. Glazińska P, Zienkiewicz A, Wojciechowski W, Kopcewicz J. The putative miR172 target gene InAPETALA2-like is involved in the photoperiodic flower induction of Ipomoea nil. J Plant Physiol. 2009; 166(16), 1801-1813.

19. Allen E, Xie Z, Gustafson AM., Sung GH, Spatafora JW, Carrington JC. Evolution of microRNA genes by inverted duplication of target gene sequences in Arabidopsis thaliana. Nat Genet. 2004; 36(12): 1282-1290.

20. Fahlgren N, Howell MD, Kasschau KD, Chapman EJ, Sullivan CM, Cumbie JS, Givan SA, Law TF, Grant SR, Dangl JL, et al. Highthroughput sequencing of Arabidopsis microRNAs: evidence for frequent birth and death of MIRNA genes. PLoS One. $2007 ; 2(2)$ : e219.

21. Hou G, Du CY, Gao HH, Liu SJ, Sun W, Lu HF, Kang J, Xie YX, Ma DY, Wang CY. Identification of microRNAs in developing wheat grain that are potentially involved in regulating grain characteristics and the response to nitrogen levels. BMC Plant Biol. 2020; 20(1): 87.

22. Naik HK, Devaraj VR. Genomic identification of salt induced microRNAs in niger (Guizotia abyssinica). Plant Gene, 2020; 23 : 100242.

23. Liu J, Guo X, Zhai T, Shu A, Zhao L, Liu Z, Zhang S. Genome-wide identification and characterization of microRNAs responding to ABA and GA in maize embryos during seed germination. Plant Biol (Stuttg). 2020; 22(5): 949-957.

24. Peng HS, Liu WZ, Hu ZH. Advances in studies on biology and chemical constituents in Radix Pseudostellariae. Chinese Traditional and Herbal Drugs. 2008; 37: 952-956.

25. Wong CK, Leung KN, Fung KP, Pang PK, Choy YM. Mitogenic and tumor necrosis factor producing activities of Pseudostellaria heterophylla. Int J Immunopharmacol. 1992; 14(8): 1315-1320.

26. Sheng R, Xu XX, Tang Q, Bian DF, Li Y, Qian C, He X, Gao XH, Pan R, Wang C, et al. Polysaccharide of radix Pseudostellariaeimproves chronic fatigue syndrome induced by poly I:C in mice. Evid Based Complement Alternat Med. 2009; 2011(12): 840516.

Page 10/21 
27. Pang WS, Lin SD, Dai QW, Zhang HC, Hu J. Antitussive activity of Pseudostellaria heterophylla (Miq.) Pax extracts and improvement in lung function via adjustment of multi-cytokine levels. Molecules. 2011; 16(4): 3360-3370.

28. Li J, Zhen W., Long DK, Ding L, Gong AH, Xiao CH, Jiang WK, Liu XQ, Zhou T, Huang LQ. De Novo sequencing and assembly analysis of the Pseudostellaria heterophylla PLoS One. 2016; 11(10): e0164235.

29. Xu XW, Wang KX, Pan JW, Chen XH. Small RNA sequencing identifies cucumber miRNA roles in waterlogging-triggered adventitious root primordia formation. Mol Biol Rep. 2019; 46(6): 6381-6389.

30. Jin WB, Li NN, Zhang B, Wu FL, Li WJ, Guo AG, Deng ZY. Identification and verification of microRNA in wheat (Triticum aestivum). J Plant Res. 2008; 121(3): 351-355.

31. Zuker M. Mfold web server for nucleic acid folding and hybridization prediction. Nucleic Acids Res. 2003; 31(13): 3406-3415.

32. Zhang BH, Pan XP, Cobb GP, Anderson TA. Plant microRNA: a small regulatory molecule with big impact. Dev Biol. 2006; 289 (1): 3-16.

33. Guo HS, Xie Q, Fei JF, Chua NH. MicroRNA directs mRNA cleavage of the transcription factor NAC1 to downregulate auxin signals for Arabidopsis lateral root development. Plant Cell. 2005; 17(5): 1376-1386.

34. Wang JW, Wang LJ, Mao YB, Cai WJ, Xue HW, Chen XY. Control of root cap formation by MicroRNA-targeted auxin response factors in Arabidopsis. Plant Cell. 2005; 17(8): 2204-2216.

35. Zhao B, Ge L, Liang R, Li W, Ruan K, Lin H, Jin Y. Members of miR-169 family are induced by high salinity and transiently inhibit the NFYA transcription factor. BMC Mol Biol. 2009; 10: 29.

36. Yang CH, Li DY, Mao DH, Liu X, Ji CJ, Li XB, Zhao XF, Cheng ZK, Chen CY, Zhu LH. Overexpression of microRNA319 impacts leaf morphogenesis and leads to enhanced cold tolerance in rice (Oryza sativa). Plant Cell Environ. 2013; 36(12): $2207-2218$.

37. Silva EMD, Silva GFFE, Bidoia DB, Silva D, Azevedo M, Jesus FAD, Pino LE, Peres LEP, Carrera E, López-Díaz I, et al. microRNA159targeted SIGAMYB transcription factors are required for fruit set in tomato. Plant J. 2017; 92(1): 95-109.

38. Li XY, Hou YM, Xie X, Li HX, Li XD, Zhu Y, Zhai LL, Zhang CY, Bian SM. A blueberry MIR156a-SPL12 module coordinates the accumulation of chlorophylls and anthocyanins during fruit ripening. J Exp Bot. 2020; 71(19): 5976-5989.

39. Navarro C, Abelenda JA, Cruz-Oró E, Cuéllar CA, Tamaki S, Silva J, Shimamoto K; Prat S. Control of flowering and storage organ formation in potato by FLOWERING LOCUS T. Nature. 2011; 478(7367): 119-122.

40. Ye ZY, Ruan SJ, Wang YY, Tian HQ. Micro-root tuber development and histochemistry study of Pseudostellaria heterophylla. Plant Physiol Commun. 2009; 45: 981-985.

41. Reinhart BJ, Weinstein EG, Rhoades MW, Bartel B, Bartel DP. MicroRNAs in plants. Genes Dev. 2002; 16(13): $1616-1626$.

42. Llave C, Xie Z, Kasschau KD, Carrington JC. Cleavage of Scarecrow-like mRNA targets directed by a class of Arabidopsis Science. 2002; 297(5589): 2053-2056.

43. Helariutta Y, Fukaki H, Wysocka-Diller J, Nakajima K, Jung J, Sena G, Hauser MT, Benfey PN. The SHORT-ROOT gene controls radial patterning of the Arabidopsis root through radial signaling. Cell. 2000; 101(5): 555-567.

44. Silverstone AL, Ciampaglio CN, Sun T. The Arabidopsis RGA gene encodes a transcriptional regulator repressing the gibberellin signal transduction pathway. Plant Cell. 1998; 10(2): 155-169.

45. Li ZX, Li SG, Zhang LF, Han SY, Li WF, Xu HY, Yang WH, Liu YL, Fan YR, Qi Over-expression of miR166a inhibits cotyledon formation in somatic embryos and promotes lateral root development in seedlings of Larix leptolepis. Plant Cell Tiss Organ Cult.2016; 127: 461-473.

46. Mallory AC, Bartel DP, Bartel B. MicroRNA-directed regulation of Arabidopsis AUXIN RESPONSE FACTOR17 is essential for proper development and modulates expression of early auxin response genes. Plant Cell. 2005; 17(5): 1360-1375.

47. Lu C, Fedoroff N. A mutation in the Arabidopsis HYL1 gene encoding a dsRNA binding protein affects responses to abscisic acid, auxin, and cytokinin. Plant Cell. 2000; 12(12): 2351-2366.

48. Xie Q, Frugis G, Colgan D, Chua NH. Arabidopsis NAC1 transduces auxin signal downstream of TIR1 to promote lateral root development. Genes Dev. 2000; 14(23): 3024-3036.

49. Feng XM, Qiao Y, Wang SS, Hao YJ. Overexpression of Arabidopsis AtmiR393a gene alters the sensitivity to auxin in tomato. Sci Hortic. 2010; 124(2): 165-169.

50. Nagpal P, Ellis CM, Weber H, Ploense SE, Barkawi LS, Guilfoyle TJ, Hagen G, Alonso JM, Cohen JD, Farmer EE, et al. Auxin response factors ARF6 and ARF8 promote jasmonic acid production and flower maturation. Development. 2005; 13(18): 4107-4118.

51. Gutierrez L, Bussell JD, Pacurar DI., Schwambach J, Pacurar M, Bellini C. Phenotypic plasticity of adventitious rooting in Arabidopsis is controlled by complex regulation of AUXIN RESPONSE FACTOR transcripts and microRNA abundance. Plant Cell. 2009; 21(10): 31193132. 
52. Matsuo T, Mitsuzono H, Okada R, Itoo S. Variations in the levels of major free cytokinins and free abscisic acid during tuber development of sweet potato. J Plant Growth Regul.1988; 7(4): 249-258.

53. Takahashi K, Fujino K, Kikuta Y, Koda Y. Expansion of potato cells in response to jasmonic acid. Plant Sci.1994; 100(1): 3-8.

54. Shi CY, Wang ZL, Guo FG, Yu SL. Changes of ATPase activity, ATP and ABA content in storage roots during storage root chickening of sweet potato. Acta Bot Boreal-Occident Sin.2002; 22(2): 315-320.

55. Robert-Seilaniantz A, MacLean D, Jikumaru Y, Hill L, Yamaguchi S, Kamiya Y, Jones JD. The microRNA miR393 re-directs secondary metabolite biosynthesis away from camalexin and towards glucosinolates. Plant J. 2011; 67(2): 218-231.

56. Yin KQ, Tang Y, Zhao JP. Genome-Wide Characterization of miRNAs involved in N gene-mediated immunity in response to Tobacco Mosaic Virus in Nicotiana benthamiana. Evol Bioinform Online. 2015; 11(Suppl 1): 1-11.

57. Guo WX, Wu GT, Yan F, Lu YW, Zheng HY, Lin L, Chen HR, Chen JP. Identification of novel Oryza sativa miRNAs in deep sequencingbased small RNA libraries of rice infected with Rice stripe virus. PLoS One. 2012; 7(10): e46443.

58. Batista PJ, Chang HY. Long noncoding RNAs: cellular address codes in development and disease. Cell. 2013; $152(6)$ : $1298-1307$.

59. Yoon JH, Abdelmohsen K, Gorospe M. Functional interactions among microRNAs and long noncoding RNAs. Semin Cell Dev Biol. 2014; 34: 9-14.

60. Ørom UA, Shiekhattar R. Long non-coding RNAs and enhancers. Curr Opin Genet Dev. 2011; 21(2): $194-198$.

\section{Figures}

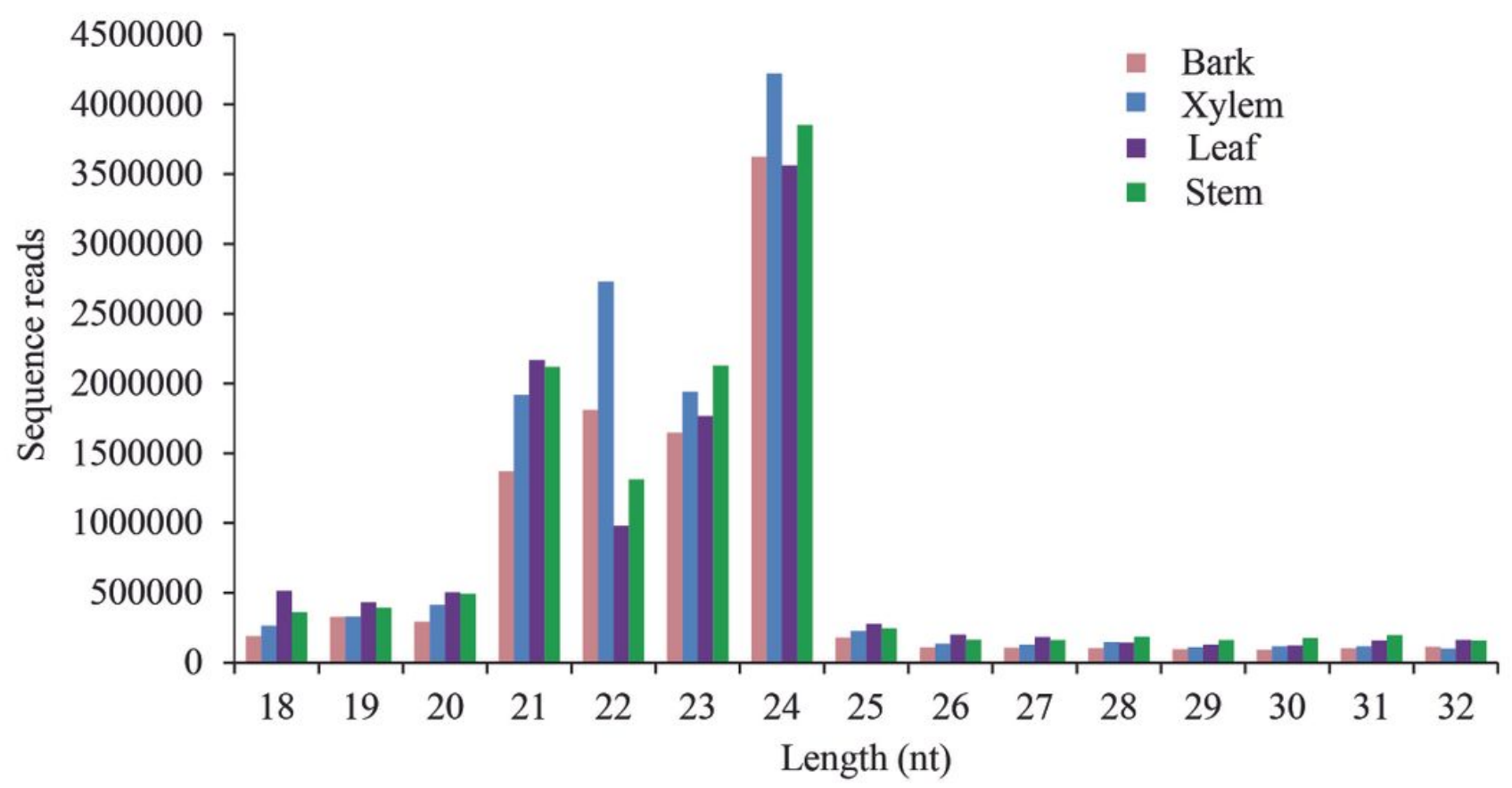

Figure 1

Size distribution of sRNAs in the leaves, stem, tuberous root bark and xylem of P. heterophylla. nt, nucleotides. 


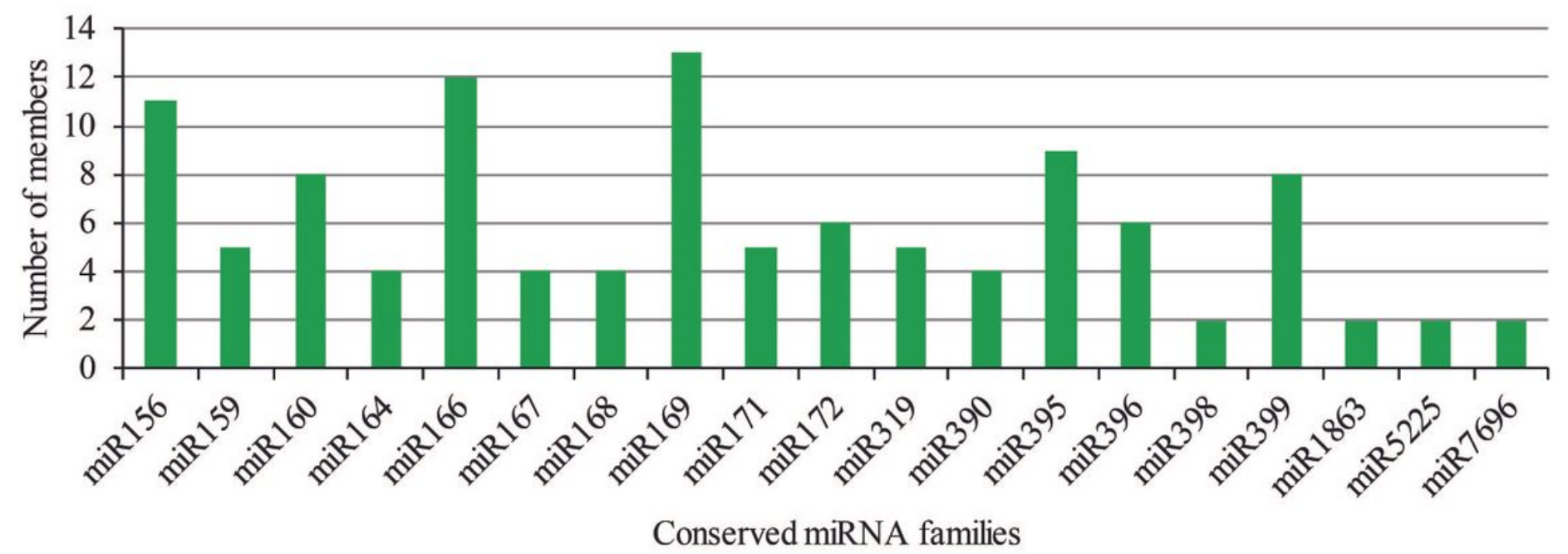

Figure 2

Conserved miRNA families and their members identified in P. heterophylla. Graphical representation of the different members of conserved miRNA families identified by deep sequencing of small RNA libraries prepared from tissues of leaves, stem, tuberous root bark and xylem of P. heterophylla.

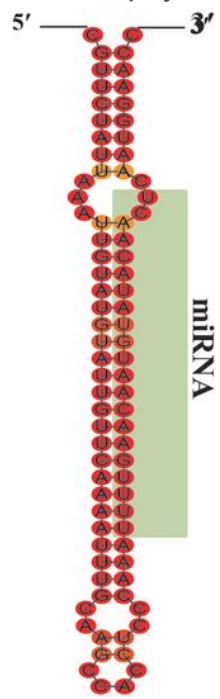

Nov-m0024

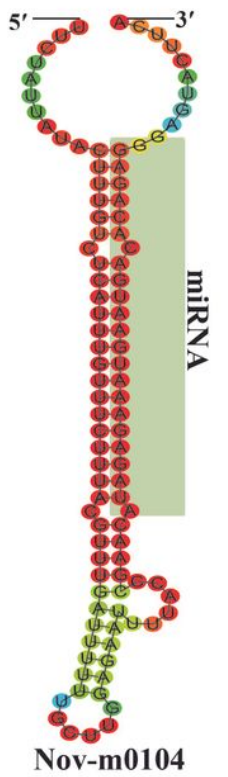

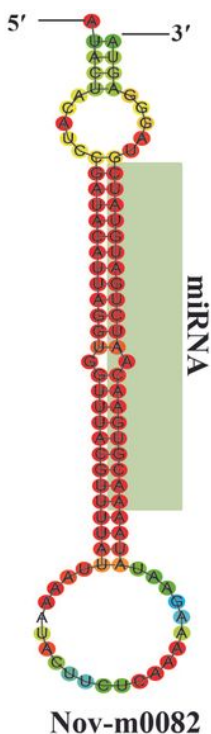

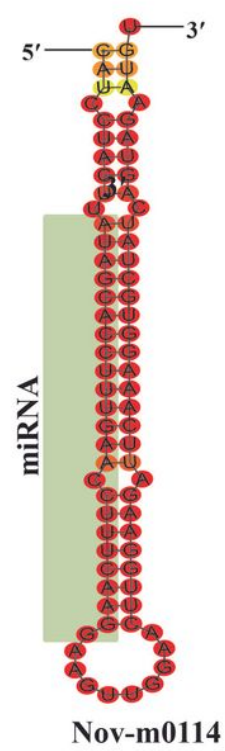

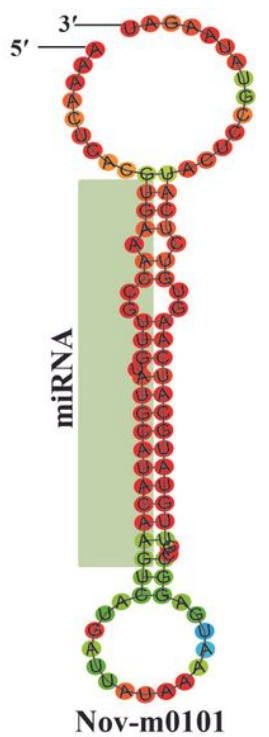

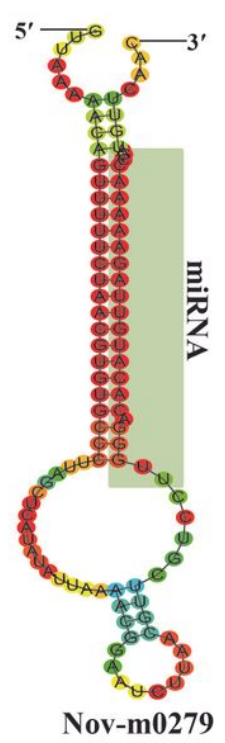


Figure 3

Predicted secondary structure of precursors (Mfold) of few specific miRNAs of P. heterophylla. The mature miRNA sequences are highlighted in light green colour.

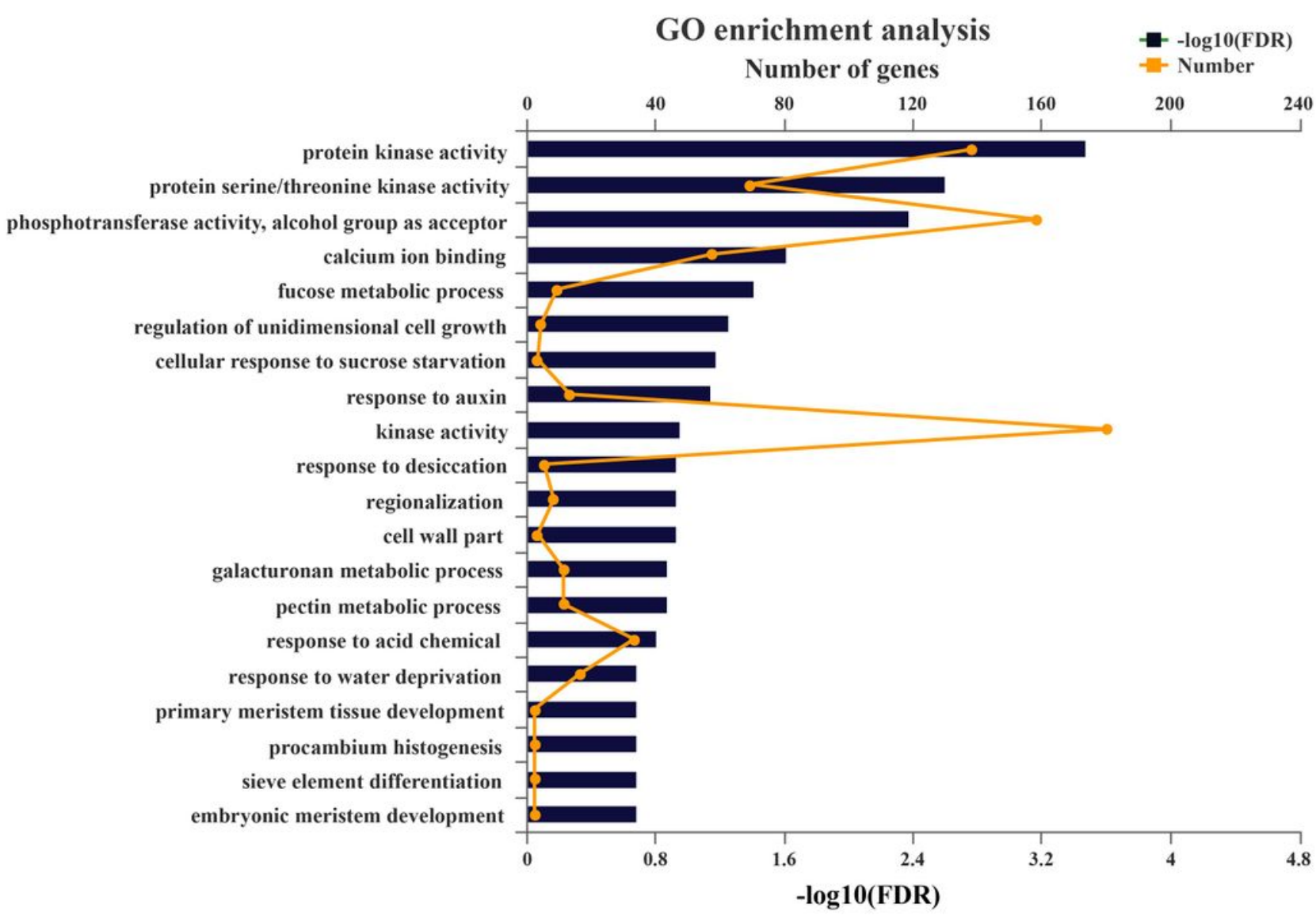

Figure 4

GO analysis of predicted putative target transcripts of miRNAs. The number of matched unigenes by the broken line. The significance of the matched gene ratio was shown by column length of FDR. 


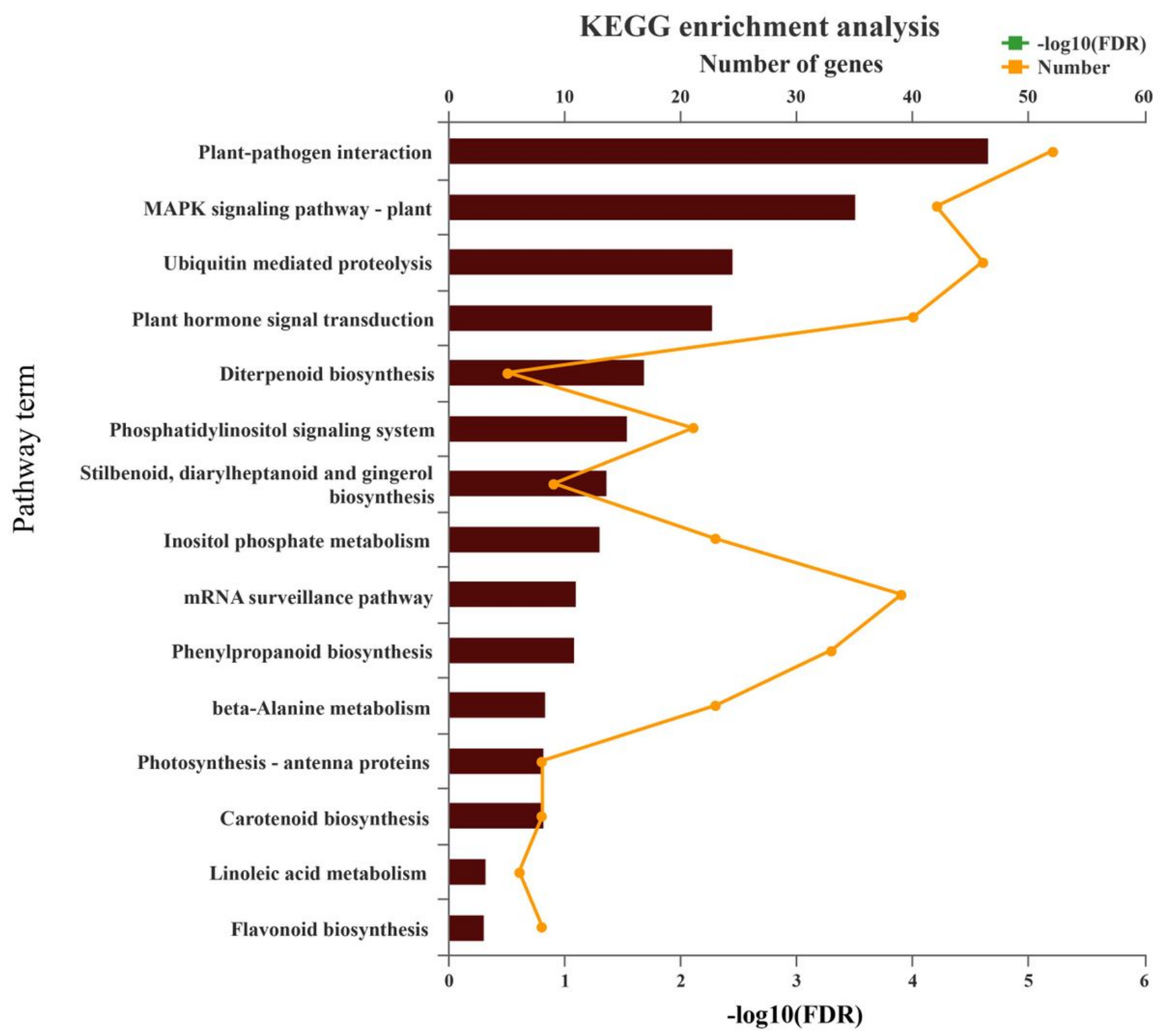

\section{Figure 5}

KEGG analysis of pathway enrichment for predicted putative target transcripts of miRNAs in the P. heterophylla. The number of matched unigenes by the broken line. The significance of the matched gene ratio was shown by column length of FDR. 

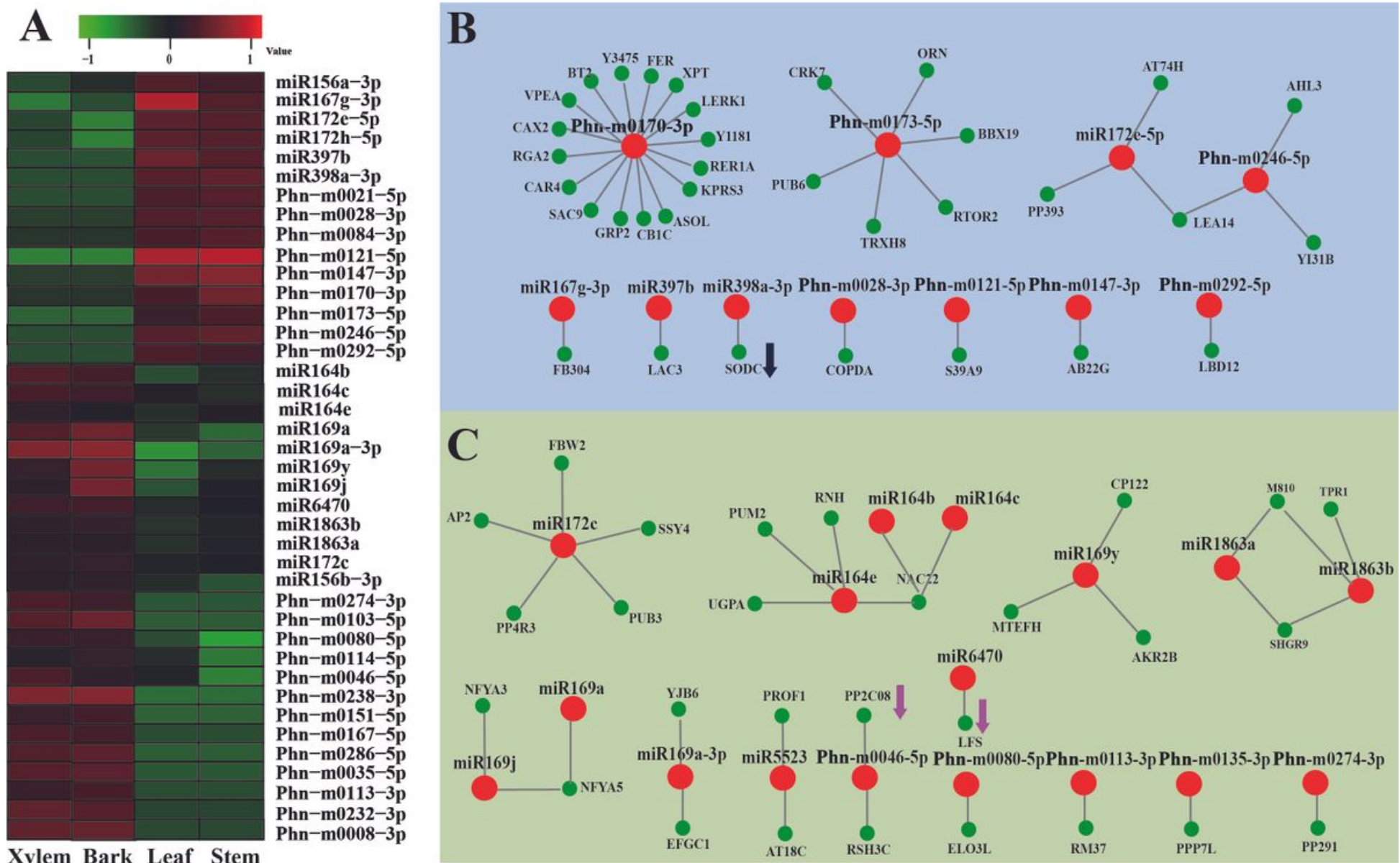

Xylem Bark Leaf Stem

\section{Figure 6}

Tissue-special miRNAs in ground parts and underground parts and their target gene. (A) Hot-map revealed the changes of miRNAs in ground parts and underground parts; (B) A combined view of reverse expression between miRNA and its target in ground parts, black tip represent significant negative correlation; (C) A combined view of reverse expression between miRNA and its target in underground parts, pink tip represent significant negative correlation. 

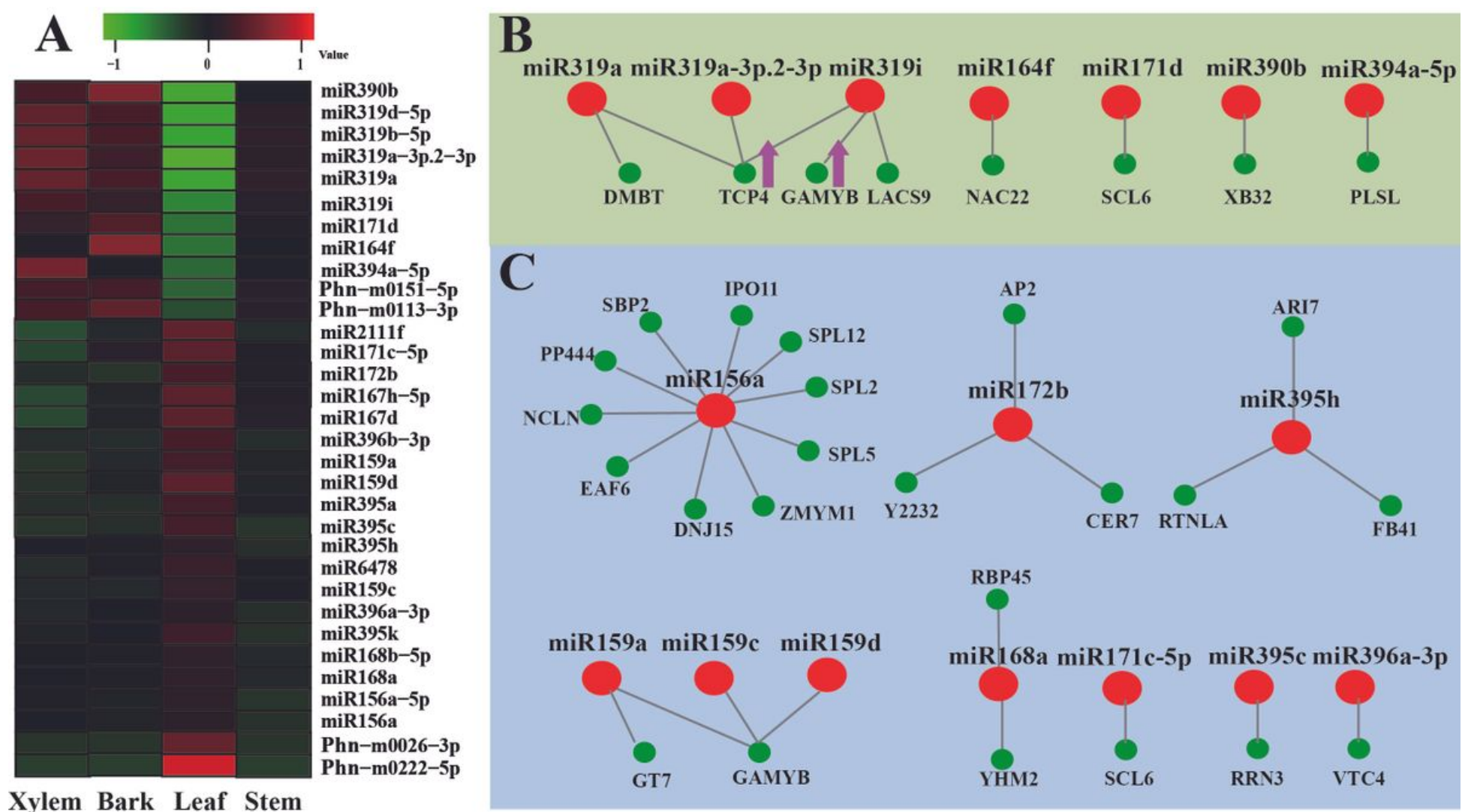

Xylem Bark Leaf Stem

\section{Figure 7}

Leaf-special miRNAs and their target gene (A) Hot-map revealed the leaf-special miRNAs; (B) A combined view of down-regulated miRNAs and its target, pink tip represent significant negative correlation; (C) A combined view of up-regulted miRNAs and its target.
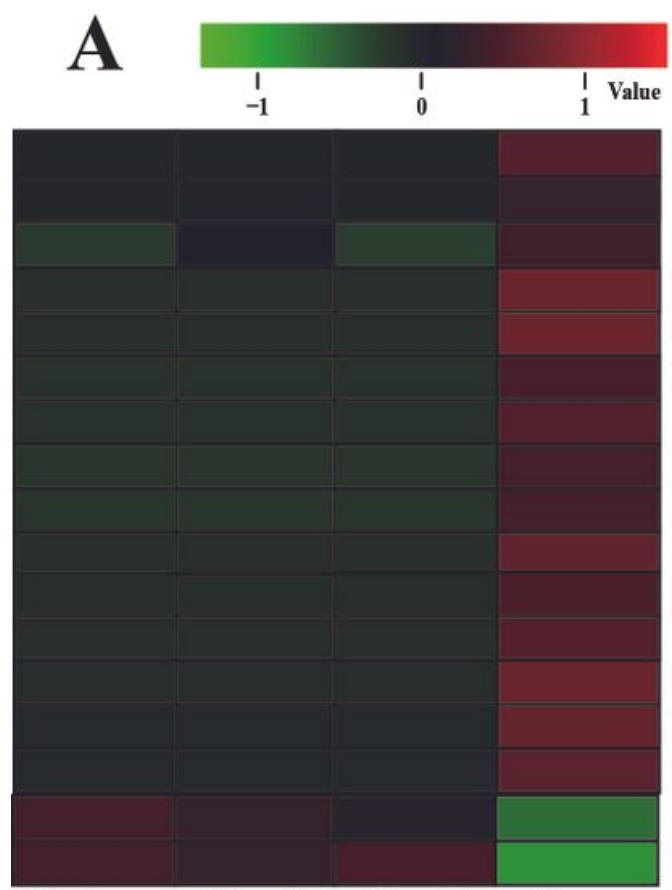

\section{Xylem Bark Leaf Stem}

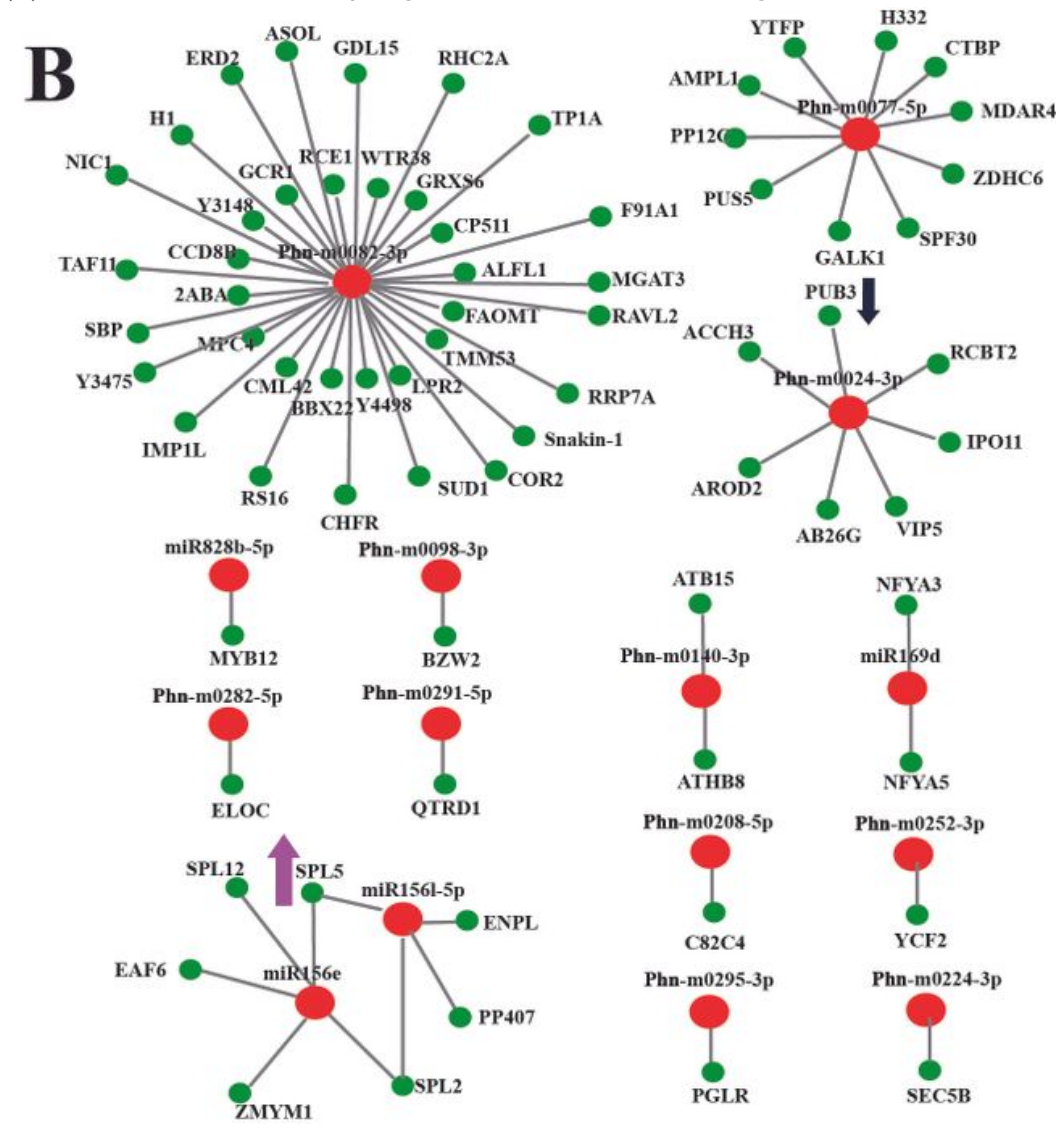

miR828b-5p

miR169d

miR156e

Phn-m0282-5p

Phn-m0082-3p

Phn-m0291-5p

Phn-m0077-5p

Phn-m0252-3p

Phn-m0038-5p

Phn-m0295-3p

Phn-m0044-5p

Phn-m0024-3p

Phn-m0140-3p

Phn-m0208-5p

Phn-m0098-3p miR1561-5p

Phn-m0224-3p

Figure 8 
Stem-special miRNAs and their target gene. (A) Hot-map revealed the stem-special miRNAs; (B) A combined view of stem-special miRNAs and its target, pink tip represent significant negative correlation between SPL5 and miR156l-5p, black tip represent significant negative correlation.
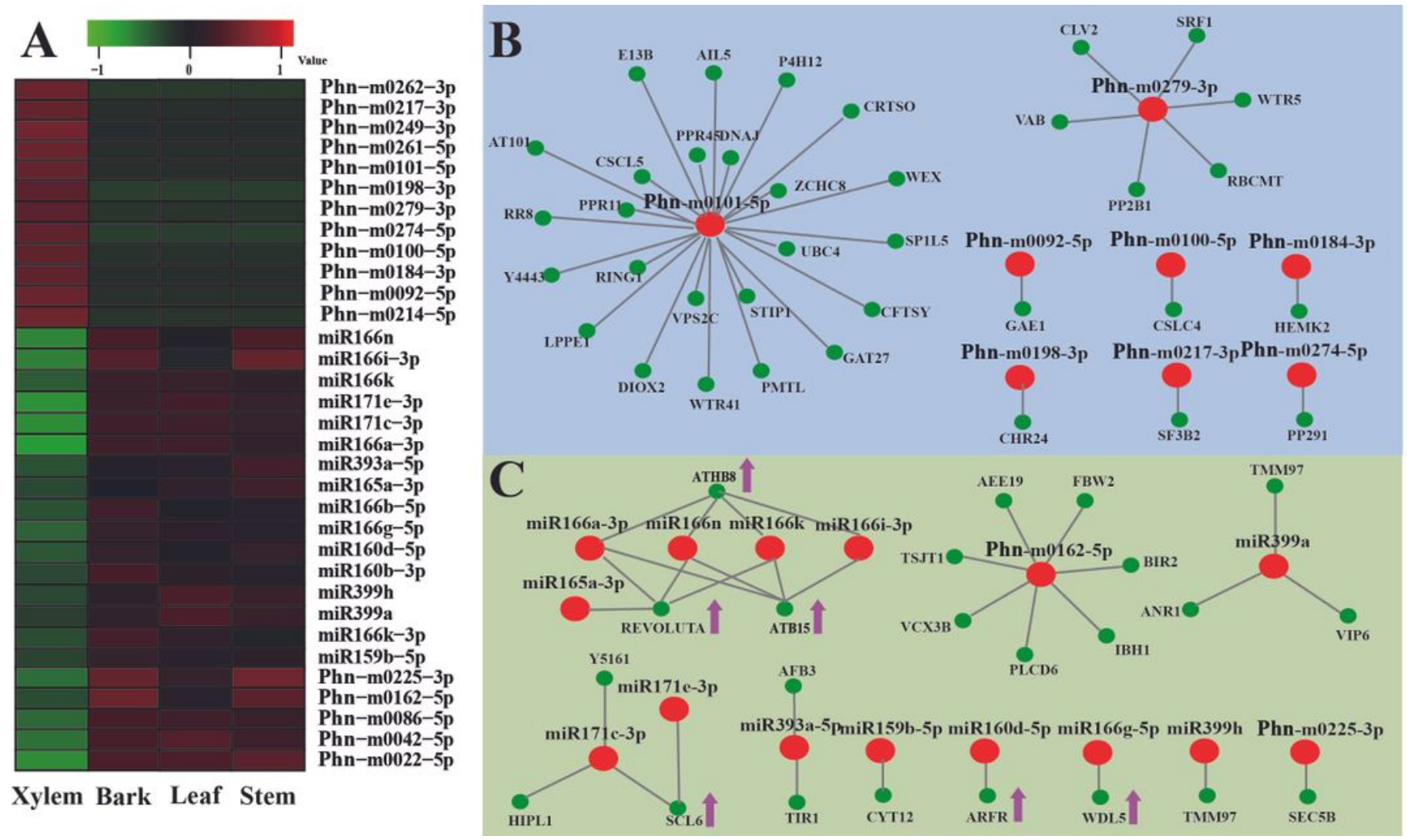

\section{Figure 9}

Xylem-special miRNAs in tuberous root and their target gene. (A) Hot-map revealed the stem-special miRNAs; (B) A combined view of upregulated miRNAs in xylem of tuberous root and its target; (C) A combined view of down-regulated miRNAs in xylem of tuberous root and its target, pink tip represent significant negative correlation between homeobox-leucine zipper protein (ATHB-8, ATHB-15 and REVOLUTA) and miR166 families, SCL6 and miR171 families, ARFR and miR160d-5p, WDL5 and miR166g-5p.

A
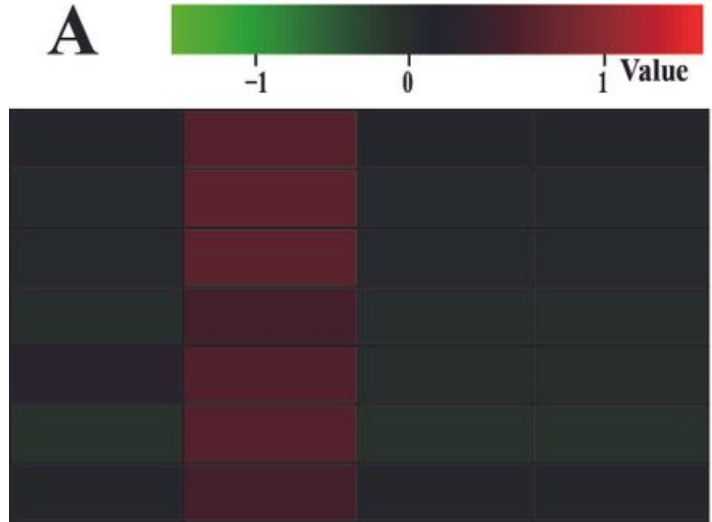

Xylem Bark Leaf Stem
B

miR160g
miR160h
miR160f-5p
miR169w
miR171a
miR390d-3p
miR390-3p

Figure 10

miR169w miR171a miR160h miR390-3p
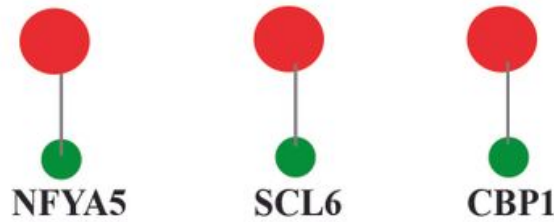

CBP1

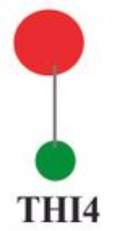

Bark-special miRNAs in tuberous root and their target gene. (A) Hot-map revealed the bark-special miRNAs; (B) A combined view of barkspecial miRNAs in xylem of tuberous root and its target. 


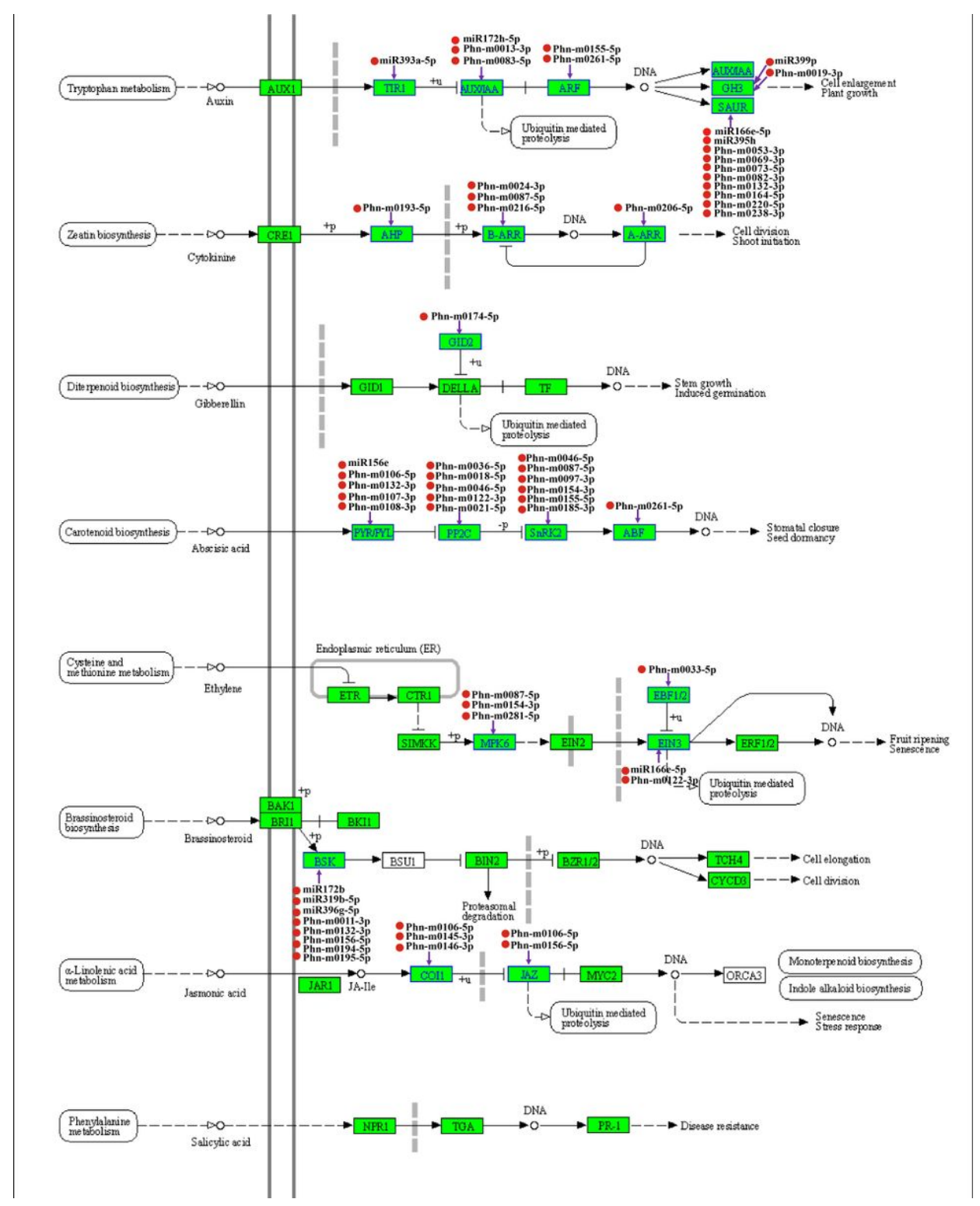

Figure 11

miRNAs and target gene in biosynthesis of plant hormone 

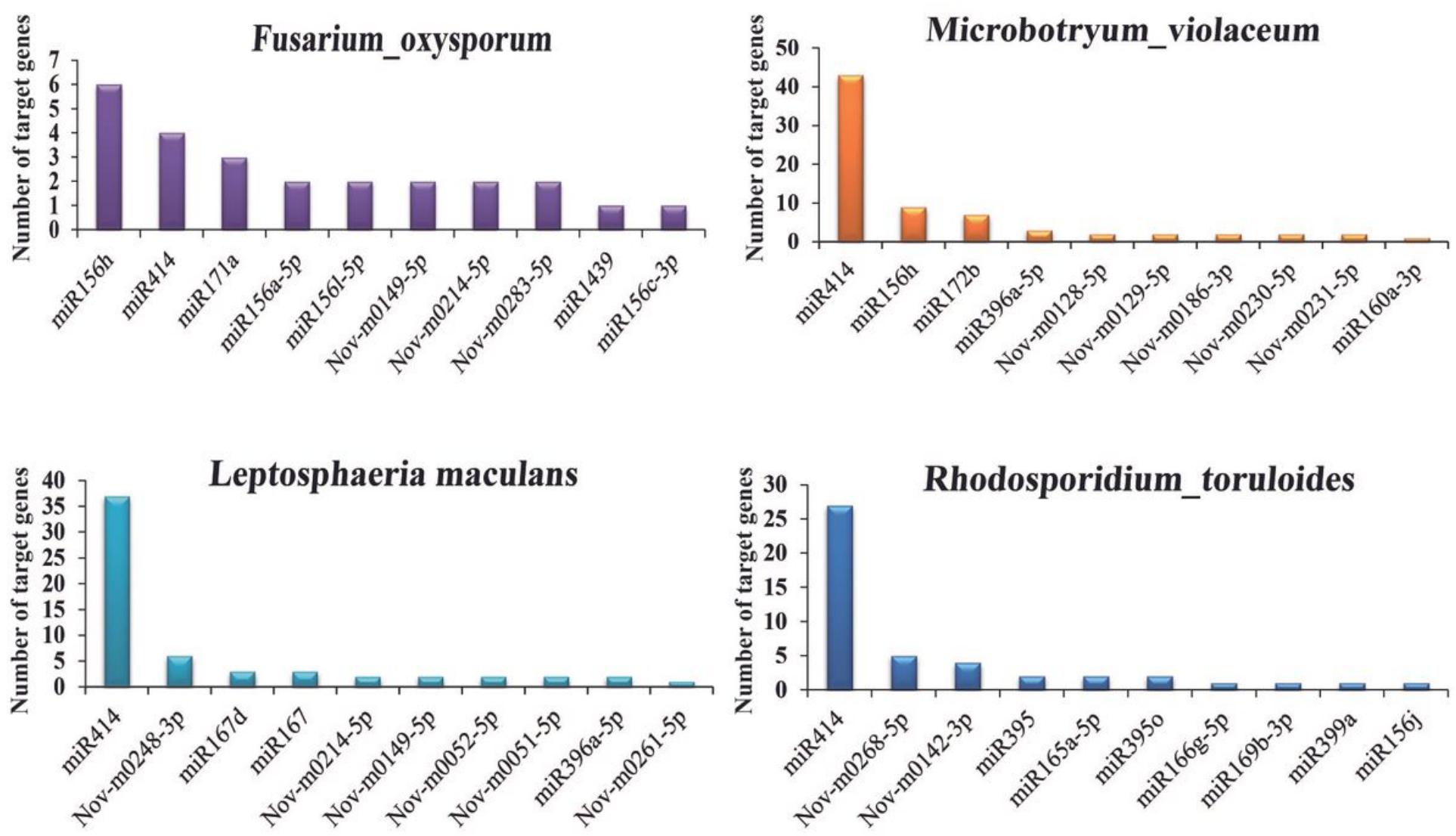

Figure 12

Number of unigenes targeted by miRNA from P. heterophylla

HD-ZIP REVOLUTA (TRINITY_DN91558_c0_g3) $\operatorname{miR} 166 \mathrm{k}$

delta(24)-sterol reductase (TRINITY_DN86257_c0_g3)

\author{
\begin{tabular}{l|l} 
6/6 \\
AAAUGCCUGGAAUGAAGCCUGGUCCGGAUUCCAUU
\end{tabular} \\ CCCUUACUUCGGACCAGGCCU
}



Figure 13

The mRNA cleavage sites identified by 5' RLM-RACE. RLM-RACE products were identified from P. heterophylla and the 5 ' termini of mRNA fragments were labeled by the arrows. Different fraction of cloned PCR products was labeled by the numbers.

\section{Supplementary Files}

This is a list of supplementary files associated with this preprint. Click to download.

- Additionalfiles.docx

- Additionalfile1ConservedmiRNAsinP.heterophylla.xlsx

- Additionalfile2DetailedinformationofidentifiednovelmiRNAs.xlsx

- Additionalfile3TargetgeneofconservedmiRNAs.xlsx

- Additionalfile4TargetgeneofnovelmiRNAs.xlsx 
- Additionalfile5UnigenestargetedbynumbersofmiRNAs.xlsx

- Additionalfile6GOanalysisofpredictedtargetgenesofmiRNAsinP.heterophylla.xls

- Additionalfile7KEGGanalysisofpredictedtargetgenesofmiRNAsinP.heterophylla.xls

- Additionalfile8ExpressioncorrelationanalysisbetweenmiRNAsandtheirtargetgene.xls

- AdditionalfilegUpregulatedmiRNAsandtheirtargetgeneingroundpartsandundergroundmiRNAsandtheirtargetgeneinundergroundparts.xlsx

- Additionalfile10UpregulatedanddownregulatedmiRNAsandtheirtargetgeneinleaf.xlsx

- Additionalfile11UpregulatedanddownregulatedmiRNAsandtheirtargetgeneinstem.xlsx

- Additionalfile12UpregulatedanddownregulatedmiRNAsandtheirtargetgeneinxylemoftuberousroot.xlsx

- Additionalfile13UpregulatedanddownregulatedmiRNAsandtheirtargetgeneinbarkoftuberousroot.xlsx

- Additionalfile14miRNAsandtargetgeneinbiosynthesisofplanthormone.xlsx

- Additionalfile15GenefromfourendophytestargetedbymiRNAsofP.heterophylla.xlsx

- Additionalfile16PrimersusedtoamplifycleavageproductsofmiRNAtargetgenesinP.heterophyllathrough5RLMRACE.xlsx 